\title{
A novel Dock8 gene mutation confers diabetogenic susceptibility in the LEW.1AR1/Ztm-iddm rat, an animal model of human type 1 diabetes
}

\author{
Tanja Arndt ${ }^{1}$ • Dirk Wedekind ${ }^{2}$ • Anne Jörns ${ }^{1}$ - Georgios Tsiavaliaris ${ }^{3}$. \\ Edwin Cuppen $^{4} \cdot$ Hans-Jürgen Hedrich ${ }^{2} \cdot$ Sigurd Lenzen $^{1}$
}

Received: 8 May 2015 / Accepted: 24 August 2015 /Published online: 12 September 2015

(C) Springer-Verlag Berlin Heidelberg 2015

\begin{abstract}
Aims/hypothesis The LEW.1AR1-iddm rat, an animal model of human type 1 diabetes, arose through a spontaneous mutation within the inbred strain LEW.1AR1. A susceptibility locus $(\operatorname{Iddm} 8)$ on rat chromosome 1 (RNO1) has been identified previously, which is accompanied by autoimmune diabetes and the additional phenotype of a variable $\mathrm{CD}^{+} \mathrm{T}$ cell frequency.

Methods In the present study we characterised the $\operatorname{Iddm} 8$ region on RNO1 in backcross strains using the genetically divergent Brown Norway $(\mathrm{BN})$ and Paris (PAR) rats. Candidate genes of the $I d d m 8$ region were sequenced for mutation analysis.

Results The Iddm8 region could be subdivided by single nucleotide polymorphism (SNP) analyses. In the first region, a mutation in exon 44 of the Dock 8 gene was identified resulting in an amino acid exchange in the protein from glutamine to
\end{abstract}

Tanja Arndt and Dirk Wedekind contributed equally to this study.

Electronic supplementary material The online version of this article (doi:10.1007/s00125-015-3757-7) contains peer-reviewed but unedited supplementary material, which is available to authorised users.

Dirk Wedekind

wedekind.dirk@mh-hannover.de

Sigurd Lenzen

lenzen.sigurd@mh-hannover.de

Institute of Clinical Biochemistry, Hannover Medical School, 30623 Hannover, Germany

2 Institute for Laboratory Animal Science, Hannover Medical School, 30623 Hannover, Germany

3 Institute for Biophysical Chemistry, Hannover Medical School, Hannover, Germany

4 Centre for Biomedical Genetics, Hubrecht Institute, Utrecht, The Netherlands glutamate. This exchange is unique for the LEW.1AR1-iddm rat. In the second region, a SNP was detected in exon 11 of the $V w a 2$ gene with an exchange from arginine to tryptophan. This SNP is also present in other rat strains.

Conclusions/interpretation The Dock8 mutation gave rise to a new type 1 diabetes rat model with very close similarity to type 1 diabetes in humans, providing a deepened insight into the impact of genes involved in diabetes development.

Keywords Animal model $\cdot$ Mutation $\cdot$ Tcells $\cdot$ Type 1 diabetes

\author{
Abbreviations \\ $\mathrm{BB}$ rat BioBreeding diabetes-prone rat \\ BN Brown Norway \\ BN N2 $([\mathrm{BN} \times$ LEW.1AR1-iddm $] \times$ LEW.1AR1-iddm $)$ N2 \\ DHR Dedicator of cytokinesis homology region \\ DOCK Dedicator of cytokinesis \\ GEF Guanine nucleotide exchange factor \\ NKT Natural killer T \\ PAR Paris rat \\ PAR N2 $([$ PAR $\times$ LEW.1AR1-iddm $] \times$ LEW.1AR1-iddm $)$ \\ $\mathrm{N} 2$ \\ RNO1 Rat chromosome 1 \\ SNP Single nucleotide polymorphism \\ VWA von Willebrand factor A domain containing
}

\section{Introduction}

Animal models play an important role for the understanding of the pathogenesis as well as the genetics of type 1 diabetes since they provide the opportunity to combine genetic and functional characterisation of the syndrome [1-3]. The 
LEW.1AR1/Ztm-iddm rat is a model of human type 1 diabetes, which arose through a spontaneous mutation in the intraMHC recombinant inbred strain LEW.1AR1 in 1997 [4]. This diabetes syndrome is of a proven autoimmune nature $[5,6]$ leading to apoptotic beta cell destruction, induced by proinflammatory cytokines released from islet infiltrating immune cells $[7,8]$.

The mode of inheritance is autosomal recessive with an incomplete penetrance of the mutant phenotype of about $60 \%[7,8]$. In a previous study in the LEW.1AR1-iddm model, three type 1 diabetes susceptibility loci have been discovered by linkage analysis using a Brown Norway (BN) backcross population (BN N2) [9].

Out of these identified three loci, one locus could be mapped to RNO20p12 within the MHC II region that also provides type 1 diabetes susceptibility in humans (IDDMI [also known as $H L A-D Q B 1]$ ), the NOD mouse (Idd1), and the BioBreeding diabetes-prone $(\mathrm{BB})$ and the Komeda rat models (Iddm1) [10]. Thus, the MHC II haplotype plays a pivotal role in permitting type 1 diabetes development [11].

The other two Iddm loci reside on rat chromosome 1 (RNO1) in the LEW.1AR1-iddm rat [9]. The Iddm8 locus was discovered within RNO1q51-55 at the telomeric end and $\operatorname{Iddm} 9$ could be localised in RNO1p11-1q11 near the centromere using the BN N2 backcross population [9]. In an additional backcross population with the Paris rat (PAR) strain (PAR N2) the Iddm1 and Iddm8 loci could be confirmed [12].

The Iddm8 locus could also be associated with a second phenotype in addition to type 1 diabetes development, described as a variable $\mathrm{CD}^{+} \mathrm{T}$ cell frequency in peripheral blood lymphocytes in the LEW.1AR1-iddm rat [13].

The aim of this study was to fine map the $I d d m 8$ region and to identify the mutation that is accompanied by autoimmune diabetes development and a variable $\mathrm{CD}^{+}{ }^{+}$T cell frequency in the LEW.1AR1-iddm rat.

\section{Methods}

Animals To identify, in the LEW.1AR1/Ztm-iddm rat, a susceptibility locus of the mutation going along with diabetes, a ([LEW.1AR1-iddm $\times$ PAR] $\times$ LEW.1AR1-iddm) backcross population (PAR N2, diabetes incidence $\sim 6.5 \%$ ) and a ([LEW.1AR1-iddm $\times$ BN] $\times$ LEW.1AR1-iddm) backcross population (BN N2, diabetes incidence $\sim 16 \%$ ) were generated as described previously [9, 12]. Diabetic BN and PAR N2 animals were analysed for their genetic characteristics [9, 12]. To prove the impact of the identified mutation, an F2 colony was generated by mating F1 (LEW.1AR $1 \times$ LEW.1AR1-iddm) animals. The distribution of the genotypes in a (LEW.1AR1 $\times$ LEW.1AR1-iddm) F2 generation according to Mendelian rules is $25 \%$ homozygous for the wild-type, $25 \%$ homozygous for the mutation and $50 \%$ heterozygous.
Because the mutation in the LEW.1AR1-iddm rat is autosomal recessive, only animals that are homozygous for the mutation become diabetic.

All animals were bred under specified pathogen free conditions and later housed together in the same hygienic unit in the Central Animal Facility of Hannover Medical School (Ztm) [13]. Blood glucose was measured by the glucose oxidase method (Glucometer Elite, Bayer, Leverkusen, Germany) in blood taken from the tail vein. Diabetic animals were killed within $48 \mathrm{~h}$ after onset of hyperglycaemia ( $\geq 10 \mathrm{mmol} / \mathrm{l})$. Autoimmune diabetes was confirmed morphologically in each pancreas by diagnosis of an islet immune cell infiltration [7]. The same procedure was applied to non-diabetic animals at the age of 120 days. Experimental procedures were performed according to the German Animal Welfare Act and approved by the Local Institutional Animal Care and Research Advisory Committee of Hannover Medical School and the Lower Saxony State Office for Consumer Protection and Food Safety (Approval ID: 42500/1H).

Tissue collection Tail and ear biopsies from BN N2 $(n=218)$, PAR N2 $(n=130)$ and (LEW.1AR1 $\times$ LEW.1AR1-iddm) F2 $(n=110)$ animals were collected for preparation of genomic DNA. DNA used for genotyping of other strains, as documented in Electronic Supplementary Material (ESM) Table 1, was taken from the existing DNA library of the Central Animal Facility of Hannover Medical School. DNA from $\mathrm{BB}$ rats $(n=8)$ and Komeda rats $(n=8)$ was isolated from tissue kindly provided by A. Bone (School of Pharmacy and Biomolecular Sciences, University of Brighton, Brighton, UK) and Y. Nakaya (Institute of Health Biosciences, Department of Nutrition and Metabolism, University of Tokushima Graduate School, Tokushima, Japan), respectively. For genotyping the LEW.1AR1-iddm rat, DNA samples were used, which were collected directly after manifestation of the mutation within the background strain LEW.1AR1 (1997) as well as 5, 10 and 15 years after establishment of the inbred strain LEW.1AR1-iddm ( $n=20$ at each time point). No data were excluded because of lack of quality.

LEW.1AR1 and LEW.1AR1-iddm rats $(n=5-10$ of each strain) were killed for organ collection. Organs were frozen until RNA extraction and subsequent gene expression analyses (ESM Table 2). All experiments have been performed in triplicate.

DNA preparation Genomic DNA was extracted from the tissues using the NucleoSpin Tissue kit (Macherey-Nagel, Düren, Germany) according to the manufacturer's instructions.

Flow cytometry From all (LEW.1AR1 1 LEW.1AR1-iddm) F2 animals, blood was taken to analyse lymphocyte subpopulations in peripheral blood by flow cytometry as described 
[13]. Specifically, the natural killer T (NKT) cell population was identified by a double staining with antibodies for CD3 (G4.18) labelled with phycoerythrin (dilution 1:50; Becton Dickinson, Heidelberg, Germany) and for CD161 (NKR 10/78) labelled with fluorescein isothiocyanate (dilution 1:10, AbD Serotec, Munich, Germany). The specificity of the primary labelled antibodies was tested by isotype controls.

Single nucleotide polymorphism analyses Single nucleotide polymorphisms (SNPs) within the Iddm 8 region were selected based on new informative markers as described before [14] (CASCAD SNPview [http://cascad.niob.knaw.nl/snpview/; assessed on 10 August 2015]). All SNPs used were analysed with a specific SNP testing method from KBioscience (KASPar). SNPs were confirmed by sequencing.

Sequencing of candidate genes For sequence analysis, candidate genes were chosen according to their annotated function. For sequencing, all exons of 49 candidate genes primers were designed using Primer3 software (http://bioinfo.ut.ee/ primer3-0.4.0/; assessed on 10 August 2015). Parameters were set to design primers with an optimal melting temperature of $60^{\circ} \mathrm{C}$. All sequenced genes are listed in ESM Table 3 (including accession number from GenBank).

Structure analyses The structure of the dedicator of cytokinesis (DOCK) 8 was prepared with the PyMOL Molecular Graphics System, Version 1.5.0.4 Schrödinger LCC (Munich, Germany) using PDB-code 3VHL [15].

In situ RT-PCR Sections of the pancreas-draining lymph nodes from the (LEW.1AR1 $\times$ LEW.1AR1-iddm) F2 animals and the background strain LEW.1AR1 were placed on 3-chamber slides. The in situ RT-PCR analysis was performed on a specific thermal cycler (MJ Research, Waltham, MN, USA) as described [16]. To identify the mRNA transcripts of the mutated or wild-type Dock 8 gene, the primers showed on the $3^{\prime}$ end the specific base exchange as identified as the specific mutation in the Dock 8 gene for this animal model. The sequences of the primers are provided in ESM Table 4.

Real-time RT-PCR RNA was isolated using the RNeasy Mini Kit (Qiagen, Hilden, Germany) according to the manufacturer's instructions. Tissues were collected from LEW.1AR1 and LEW.1AR1-iddm rats and were homogenised in Isozol lysis reagent (5 Prime, Hamburg, Germany) using the Tissue Lyser LT (Qiagen) according to the manufacturer's recommendations. RNA was quantified using the NanoDrop ND-100 spectrophotometer (Thermo Scientific, Schwerte, Germany) and the quality was tested in an agarose gel. From $2 \mu \mathrm{g}$ of RNA, cDNA was synthesised using the Omniscript Reverse Transcription Kit (Qiagen) according to the manufacturer's recommendations. Sequences of the primers used for real-time RT-PCR are provided in ESM Table 5.

\section{Results}

Genetic mapping of the mutation accompanying autoimmune diabetes development in the LEW.1AR1-iddm rat $I d d m 8$ was mapped on RNO1 ( 275 genes) and is harbouring the mutation that is accompanied by diabetes development and a variable $\mathrm{CD}^{+} \mathrm{T}$ cell frequency in peripheral blood in LEW.1AR1-iddm rats (Fig. 1) [9, 12, 13].

Genotyping using SNP markers was performed in diabetic BN N2 and PAR N2 rats as well as in non-diabetic rats for both backcross strains. SNPs markers are located within the $\operatorname{Idd} m 8$ region in order to narrow down the susceptibility locus. For the BN N2 rats, 35 SNPs could be used as informative markers while for the PAR N2 rats, only 22 SNPs were usable. Two fragments in the diabetic BN N2 and one fragment in the diabetic PAR N2 could be identified within $I d d m 8$ as homozygous for LEW.1AR1-iddm markers (Fig. 1, marked in red). A more detailed analysis of the SNPs is provided in ESM Figs 1-4. All SNPs are listed in ESM Table 6 and were confirmed by sequencing with specific primers as listed in ESM Table 7. For sequence analyses 48 candidate genes were chosen according to their annotated function.

Sequencing of candidate genes Sequencing of candidate genes (ESM Table 3 ) and sequence comparison with the background strain LEW.1AR1 as well as the LEW, BN and PAR strains revealed two base exchanges leading to an amino acid exchange within the $I d d m 8$ region, one within the Dock 8 gene and one in the $V w a 2$ gene. In all other sequenced genes, including $C d 5, C d 6, P d c d 4, C a s p 7$ and Ins 1, no base exchange was detected. All sequenced genes are listed in ESM Table 3.

A mutation from $\mathrm{C}$ to $\mathrm{G}$ in Dock8 in exon 44 at position 228, 622, 763 (RGSC Genome Assembly v3.4) on RNO1 was identified, which is unique for the LEW.1AR1-iddm rat and not present in any other tested rat strain including the background strains LEW.1AR1, the LEW, BN and PAR (Fig. 2a). Dock 8 encodes for a member of the DOCK 180 protein superfamily of guanine nucleotide exchange factors (GEFs) that act as activators of Rac/Rho family GTPases [17]. The base exchange leads to an amino acid substitution from glutamine to glutamate (Q1864E) (Fig. 2b). The mutation is located in $\beta 4$ of the DOCK homology region (DHR)-2 involved in CDC42 binding [18] (Fig. 3a). The mutation may be quite critical for the function of DOCK8, since the negatively charged glutamate could form a salt bridge with the adjacent R1797 located in $\beta 1$ and interact with $\mathrm{S} 1849$, which is part of a flexible loop emerging from the $\alpha 6$ helix (Fig. 3b, red dotted lines). The loop itself contains residues that are involved in CDC42 binding [18]. The changes in number and strength of electrostatic 
Fig. 1 Long range physical map and non-recombination blocks of $\operatorname{Iddm} 8$. $\operatorname{Iddm} 8$ was identified by linkage analysis with microsatellite markers using the BN N2 and PAR N2 physical positions of microsatellite markers taken from previous studies $[9,12]$. The nonrecombinant blocks in Iddm8 (red boxes) were determined using SNP markers informative for the $\mathrm{BN} \mathrm{N} 2$ and PAR N2

Fig. 2 Sequencing of the Dock 8 gene. (a) Sequencing of the Dock 8 gene revealed a base exchange $[\mathrm{C} / \mathrm{G}]$ in exon 44 at position $228,622,763$ which is unique for the LEW.1AR1-iddm rat. (b) The base exchange in the Dock 8 gene leads to an amino acid exchange from glutamine to glutamate (Q1864E)

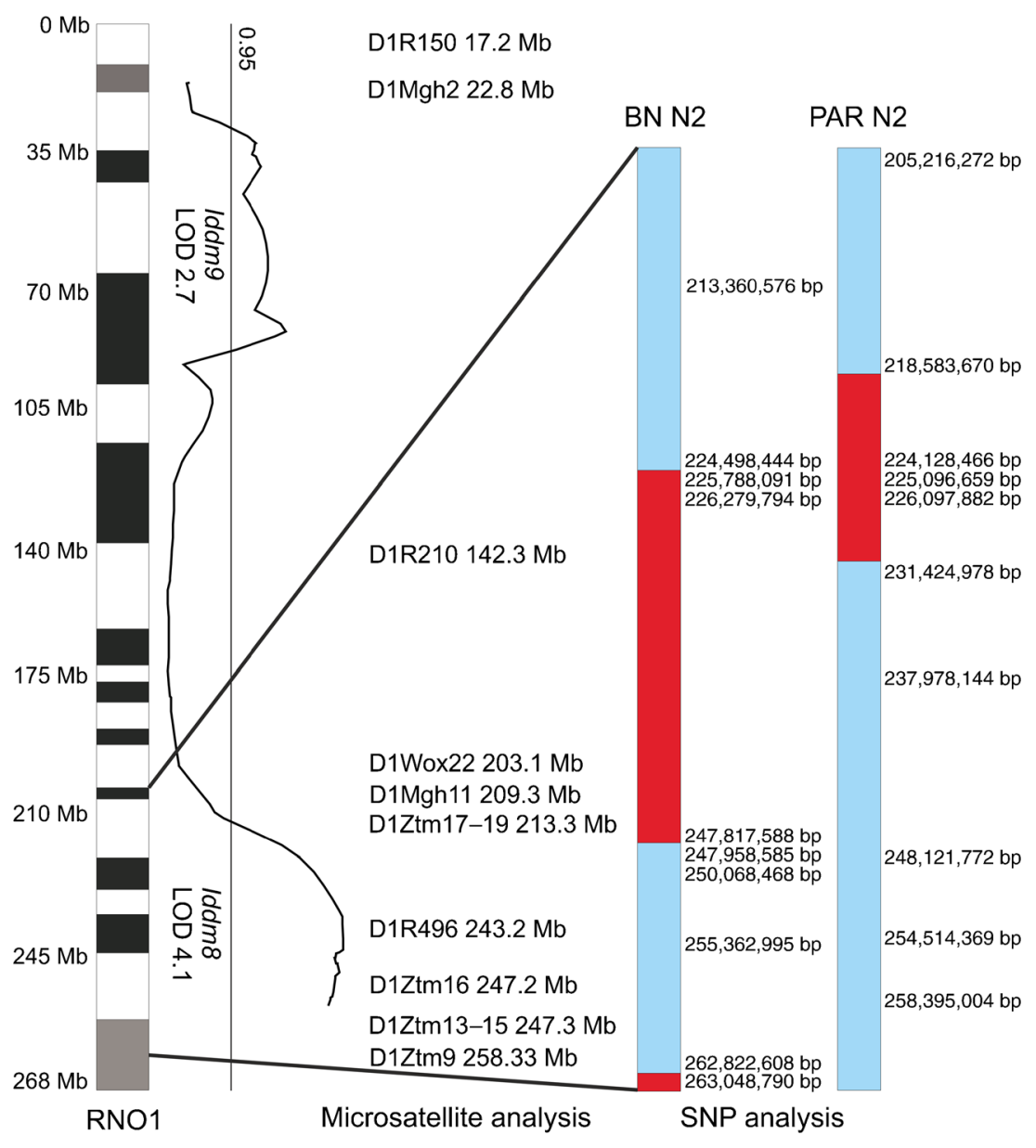

a \begin{tabular}{|l|l|l|l|l|l|l|l|l|l|l|l|}
1 & 500 & 1,000 & 1,500 & 2,000 & 2,500 & $\mid 3,000$ & 3,500 & 4,000 & 4,500 & 5,000 & 5,500 \\
\hline
\end{tabular}

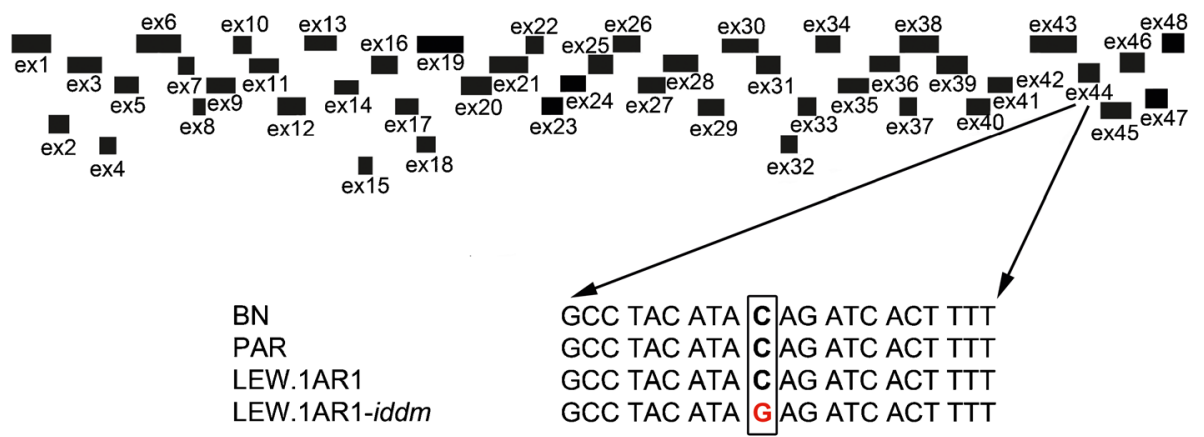

CAG (GIn) $\longrightarrow$ GAG (Glu)

b

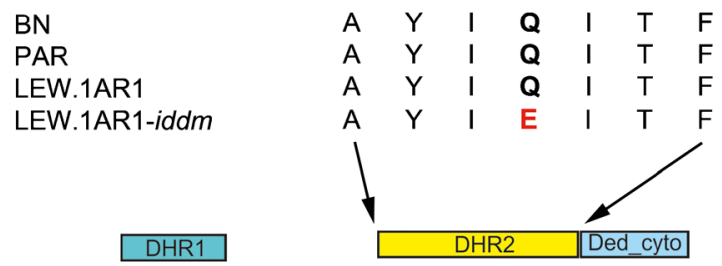




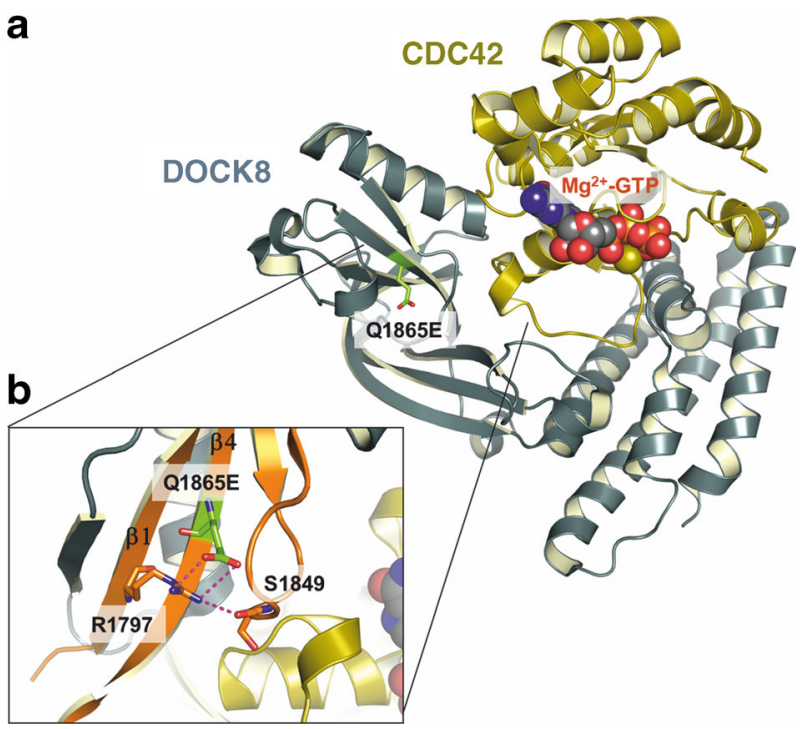

Fig. 3 Structure of the DHR2 domain of DOCK8 from mouse in complex with CDC42. (a) Mutation Q1864E in rat DOCK8 corresponds to $\mathrm{Q} 1865 \mathrm{E}$ in mouse DOCK8. The mutated amino acid is shown in green sticks and is located in the $\beta 4$-sheet of the DHR2 domain. (b) Close-up view of the area surrounding the mutation (orange). The glutamate is supposed to form a salt bridge with the adjacent arginine (R1797) of $\beta 1$ and additionally interact with residue S1849, which is part of a flexible loop emerging from the $\alpha 6$ helix. This loop is critically involved in interactions with CDC42 [18]. The proposed new interactions (red dotted lines) with surrounding residues might have an impact on the binding properties of DOCK8 with CDC42. The figure was prepared with the PyMOL Molecular Graphics System, Version 1.5.0.4 Schrödinger LCC using the PDB-code 3VHL [15]

interactions caused by the mutation most likely have an influence on the binding properties of DOCK 8 with CDC42 affecting normal function of the protein. Loss-of-function mutations in Dock 8 have been reported to cause combined immunodeficiencies [19, 20]. Another 28 different rat strains were sequenced including the two type 1 diabetes rat models, the BB rat and Komeda rat (ESM Table 1), but the Q1864E mutation was found to be unique for the LEW.1AR1-iddm rat. We analysed DNA samples from LEW.1AR1-iddm directly after occurrence of the spontaneous mutation in the background strain LEW.1AR1 as well as at later time points. The $[\mathrm{G} / \mathrm{G}]$ genotype could be verified in all these LEW.1AR-iddm rats.

In a more telomeric region, we confirmed an SNP in $V w a 2$ in exon 11. At position 263, 216, 228 (RGSC Genome Assembly v3.4) on RNO1 there was a base exchange from $\mathrm{C}$ to $\mathrm{T}$ in the LEW.1AR1-iddm rat, the LEW.1AR1 rat, the LEW rat and the PAR rat compared with the BN rat strain (Fig. 4a). This SNP changed the amino acid sequence from arginine (R681W) to tryptophan (Fig. 4b). The complete structure of von Willebrand factor A domain containing (VWA)-2 is not known; however, sequence alignments with the A2 domain of VWA2, whose high resolution structure has recently been solved [21], reveal the location of the mutation in a solventexposed loop region. We have sequenced additional rat strains and found that 17 out of 28 tested strains have the same SNP genotype (ESM Table 1). Our sequence analyses revealed that the established rat models of type 1 diabetes (LEW.1AR1$\mathrm{i} d d m$, BB, Komeda and LEW.1WR1 rats) share the same SNP genotype in exon 11 of $V w a 2$ (Table 1).

Segregation of Dock8 alleles within a (LEW.1AR1× LEW.1AR1-iddm) F2 A (LEW.1AR1 $\times$ LEW.1AR1-iddm) F2 $(n=110)$ was generated and the segregation of the Dock 8 $[\mathrm{C} / \mathrm{G}]$ alleles were analysed. From these 110 animals, 18 animals were homozygous for $[\mathrm{C} / \mathrm{C}], 60$ animals were heterozygous for $[\mathrm{C} / \mathrm{G}]$ and 32 animals were homozygous for $[\mathrm{G} / \mathrm{G}]$ (Table 2). The distribution of the genotypes is in agreement with the Mendelian rules ( $\chi^{2}$ test, $P=0.1$ ). From these 32 homozygous animals with the genotype $[\mathrm{G} / \mathrm{G}], 20$ became diabetic, corresponding to the established diabetes incidence of around $60 \%$ in the LEW.1AR1-iddm colony (Table 2) [7].

The mutation in the LEW.1AR1-iddm rat is responsible not only for diabetes development but also for a variable $\mathrm{CD}^{+} \mathrm{T}$ cell frequency in peripheral blood [13]. Therefore, we analysed the $\mathrm{CD}^{+} \mathrm{T}$ cell frequency of all $\mathrm{F} 2$ rats. The $\mathrm{CD} 3^{+}$ $\mathrm{T}$ cell frequency was slightly decreased in rats that were homozygous for the mutation $[\mathrm{G} / \mathrm{G}]$ compared with animals that were heterozygous $[\mathrm{C} / \mathrm{G}]$ or homozygous for the wild-type $[\mathrm{C} / \mathrm{C}](p<0.001$ homozygous [G/G]: $50.2 \pm 2.0 \%$ vs heterozygous $[\mathrm{C} / \mathrm{G}]: 66.0 \pm 1.2 \%$ and homozygous $[\mathrm{C} / \mathrm{C}]: 69.3 \pm 2.9 \%$ ). In parallel the $\mathrm{CV}$ was increased in animals homozygous for the mutation $[\mathrm{G} / \mathrm{G}](p<0.001$ homozygous $[\mathrm{G} / \mathrm{G}]: 22.0 \%$ vs heterozygous $[\mathrm{C} / \mathrm{G}]: 13.4 \%$ and homozygous $[\mathrm{C} / \mathrm{C}]: 12.4 \%$ ) (Table 2). The NKT frequency was slightly reduced in rats homozygous for the mutation $[\mathrm{G} / \mathrm{G}]$ compared with the wild-type $[\mathrm{C} / \mathrm{C}]$. The $\mathrm{CV}$ was not affected (Table 2).

Differentiation of Dock8 expression in immune cells using in situ RT-PCR Specific primers for the mutated Dock 8 allele and the wild-type allele were created to distinguish by in situ RT-PCR analyses the gene expression in the immune cells of the pancreas-draining lymph nodes between the (LEW.1AR1 $\times$ LEW.1AR1-iddm) F2 rats and their genetic background strain LEW.1AR1. Expression of the mutated Dock 8 mRNA transcript was observed in all rats homozygous for the mutation, while the wild-type mRNA transcript was expressed in all rats homozygous for the wild-type allele (Fig. 5).

Quantification of Dock8 expression in different tissues using real-time RT-PCR The expression of Dock 8 was analysed by real-time RT-PCR using gene specific primers located outside the mutation (spanning from exon 14 to 15). The gene expression of different tissues from LEW.1AR1$i d d m$ rats and rats from the background strain LEW.1AR1 was determined. Dock 8 gene expression was detectable in all analysed organs, even though gene expression levels were often somewhat lower in the LEW.1AR1-iddm rats compared 
Fig. 4 Sequencing of the $V w a 2$ gene. (a) Sequencing of the $V w a 2$ gene revealed a SNP $[\mathrm{C} / \mathrm{T}]$ in exon 11 at position $263,216,228$ between the $\mathrm{BN}$ rats and the LEW.1AR1-iddm, LEW.1AR1 and PAR rats. (b) The amino acid exchange is located in the third VWA domain of the protein leading to an amino acid exchange from arginine to tryptophan $(\mathrm{R} 681 \mathrm{~W})$ a \begin{tabular}{|l|l|l|l|l|l|l|l|l|l|l|l|l|}
1 & 200 & 400 & 600 & 800 & 1,000 & 1,200 & 1,400 & 1,600 & 1,800 & 2,000 & 2,200 & 2,400 \\
\hline
\end{tabular}

b

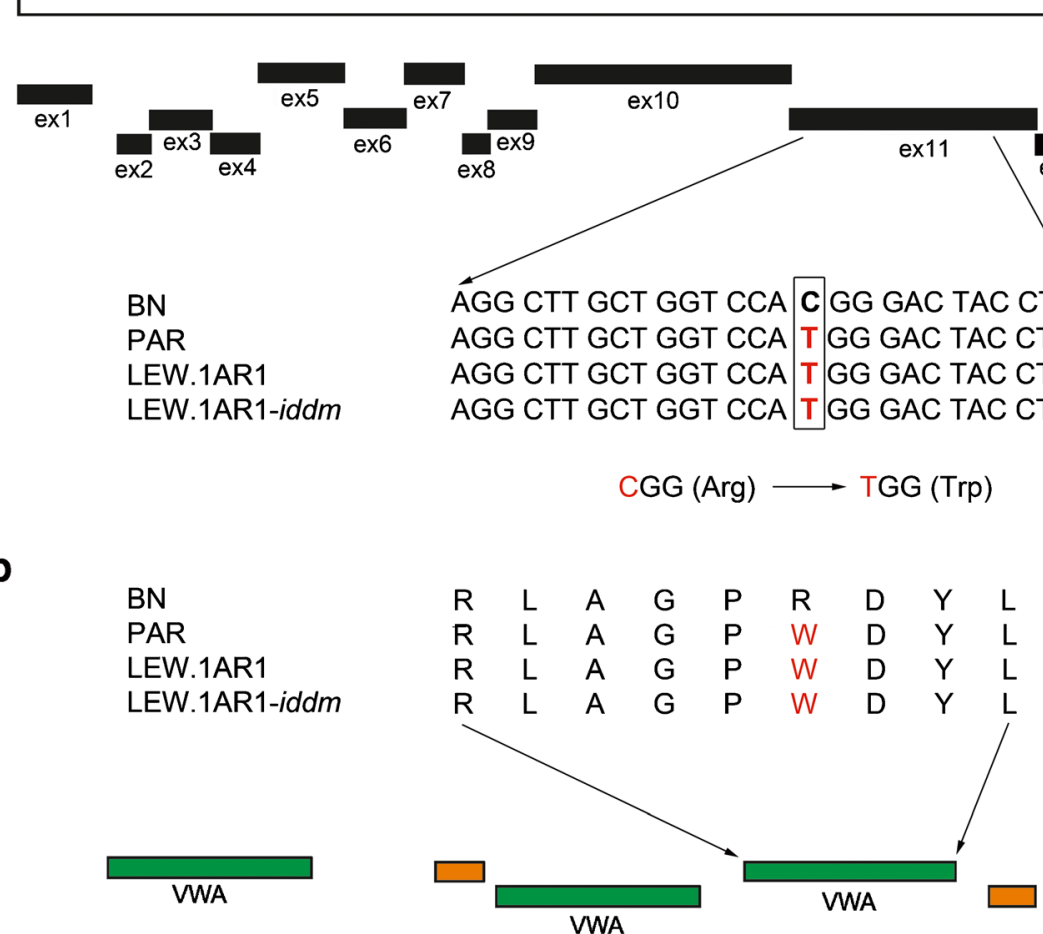

VWA

located within and outside these blocks revealed polymorphisms in the Dock8 gene at the proximal end (RNO1q51) of $I d d m 8$ and the $V w a 2$ gene at the distal end (RNO1q55) of $I d d m 8$. A base exchange $[\mathrm{C} / \mathrm{G}]$ in Dock8 is unique for the LEW.1AR1-iddm rat and leads to an amino acid exchange from glutamine to glutamate (Q1865E). A [C/T] SNP in $V w a 2$ is present in several inbred rat strains (ESM Table 1) leading to an arginine/tryptophan polymorphism (R681W). The fact, that this SNP is distributed among rat inbred strains, excluded the possibility that it is the sole spontaneous mutation in the LEW.1AR1$i d d m$ rat. Further sequence analyses revealed that all diabetes-prone rat strains such as BB and Komeda are homozygous for the $[\mathrm{T} / \mathrm{T}]$ genotype (ESM Table 1). This, however, supports the assumption that $V w a 2$ may be involved in the pathogenesis of autoimmune diabetes, as has been reported for humans [22].

Table 1 Prerequisites for diabetes development in the different type 1 diabetes rat models

\begin{tabular}{|c|c|c|c|c|}
\hline Rat model & MHC class II & Vwa2 variant & Main mutation & Diabetes \\
\hline LEW.1AR1-iddm rat & $B / D^{u}$ & $\mathrm{~T}(\operatorname{Trp})$ & $\begin{array}{l}\text { Dock8 (RNO1) } \\
\text { C (Gln) G (Glu) }\end{array}$ & Yes \\
\hline $\mathrm{BB}$ rat & $B / D^{u}$ & $\mathrm{~T}(\operatorname{Trp})$ & $\begin{array}{l}\text { Gimap5 (Ian5) (RNO4) } \\
\text { Frameshift mutation }\end{array}$ & Yes \\
\hline Komeda rat & $B / D^{u}$ & $\mathrm{~T}(\operatorname{Trp})$ & $\begin{array}{l}\text { Cblb (RNO11) } \\
\text { C (Arg) T (Stop codon) }\end{array}$ & Yes \\
\hline LEW.1WR1 rat & $B / D^{u}$ & $\mathrm{~T}(\operatorname{Trp})$ & Ubd (RNO20) & Yes \\
\hline
\end{tabular}


Table 2 Segregation of the $\mathrm{C} / \mathrm{G}$ SNP in a (LEW.1AR1 $\times$ LEW.1AR1-iddm) F2 generation

\begin{tabular}{llll}
\hline Dock8 & Homozygous [C/C] & Heterozygous [C/G] & Homozygous [G/G] \\
\hline Number of animals & 18 & 60 & 32 \\
Diabetic animals & 0 & 0 & 20 \\
Incidence (\%) & 0 & 0 & 62.5 \\
Mean CD3 ${ }^{+}(\%)$ & $69.3 \pm 2.9$ & $66.0 \pm 1.2$ & $50.2 \pm 2.0^{* * *}$ \\
CV of CD3 $^{+}(\%)$ & 12.4 & 13.4 & $22.0^{* * *}$ \\
Mean NKT (\%) & $1.8 \pm 0.1$ & $1.5 \pm 0.1$ & $1.3 \pm 1.0^{*}$ \\
CV of NKT $(\%)$ & 32.5 & 29.8 & 37.7 \\
\hline
\end{tabular}

Data are presented as mean values \pm SEM for the numbers of animals given in the table. ${ }^{*} p<0.05, * * * p<0.001$ homozygous $[\mathrm{G} / \mathrm{G}]$ vs homozygous [C/C] and heterozygous [C/G].
The segregation analysis of a (LEW.1AR1× LEW.1AR1$i d d m$ ) F2 colony confirmed the base exchange in the Dock 8 as the crucial mutation in the LEW.1AR1-iddm rat. Only rats with the $[\mathrm{G} / \mathrm{G}]$ genotype in Dock 8 were affected by a variable $\mathrm{CD}^{+} \mathrm{T}$ cell frequency of which $62 \%$ became diabetic. Additionally, the mutation was further proven by in situ RT-PCR analyses of the (LEW.1AR1 $\times$ LEW.1AR1-iddm) F2 rats.

Dock8 is well conserved between humans and rodents. Interestingly, there is not only a high conservation in Dock8 between different species but also among members of the whole Dock family [18].

DOCK 8 belongs to the DOCK180 family of atypical GEFs. All these DOCK proteins contain two characteristic domains, DHR1 and DHR2 [23]. The DHR1 domain binds to phosphatidylinositol-3,4,5-triphosphate (PtdIns[3,4,5] $\mathrm{P}_{3}$ ) and mediates the recruitment of DOCK proteins to the plasma membrane [24]. The GEF activity is located in the DHR2 domain, which binds to and activates GTPases of the Rac/ Rho family, mainly CDC42 but also Ras-related $\mathrm{C} 3$ botulinum toxin substrate 1 (RAC1) $[15,25]$. In particular, DOCK proteins regulate actin cytoskeleton, cell adhesion and migration [26]. DOCK proteins also play a role in the immune system. Dock2 deficient mice show T cell lymphopenia, a decreased cellularity of the thymus and secondary lymphatic organs, loss of the marginal zone B cells, a decreased lymphocyte chemotaxis and migration as well as a decreased $\mathrm{T}$ cell proliferation [27-30].

The mutation in the LEW.1AR1-iddm rat is located in a $\beta 4$-sheet within the DHR2 domain of the DOCK8 protein. Therefore, the mutation may have an influence on the binding capacity to GTPases. Binding to the GTPases promotes integrin reorganisation and adhesion, lamellipodia formation, cell polarisation, and phagocytosis or cell fusion [23, 31]. It is known that aberrant activation of $\mathrm{Cdc} 42$ results in pathology, such as tumourigenesis and tumour progression, cardiovascular diseases, neuronal degenerative diseases as well as diabetes [32].

DOCK 8 has already been known to play a role within the immune system. Mutations in the DOCK 8 gene were associated first with the hyper-IgE syndrome [19]. In addition to the
Fig. 5 Gene expression of Dock 8 by in situ RT-PCR. mRNA expression of the mutated and the wild-type Dock 8 gene in immune cells of pancreas-draining lymph nodes of the F2 generation from LEW.1AR1-iddm rats and the background strain LEW.1AR1 rats by in situ RT-PCR. Animals homozygous either for the mutation $[\mathrm{G} / \mathrm{G}]$ or the wild-type $[\mathrm{C} / \mathrm{C}]$ showed mRNA expression of the Dock 8 gene in immune cells using the specific primers. The animals of the background strain revealed the Dock 8 gene expression of the wild-type [C/C]. Scale bar, $25 \mu \mathrm{m} . n=4$ animals in each group
(LEW.1AR1 $\times$ LEW.1AR1-iddm) F2 generation

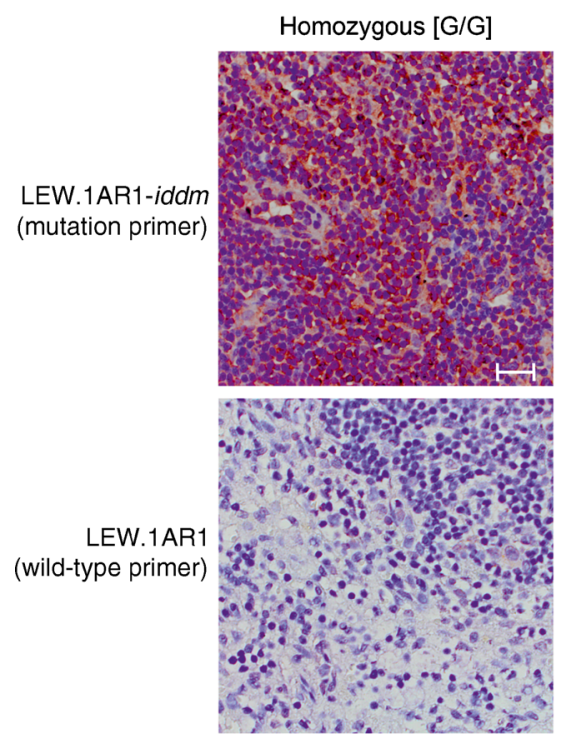

LEW.1AR1 background

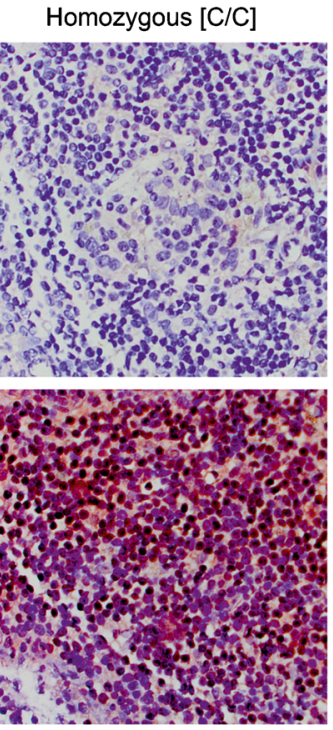

Homozygous $[\mathrm{C} / \mathrm{C}]$

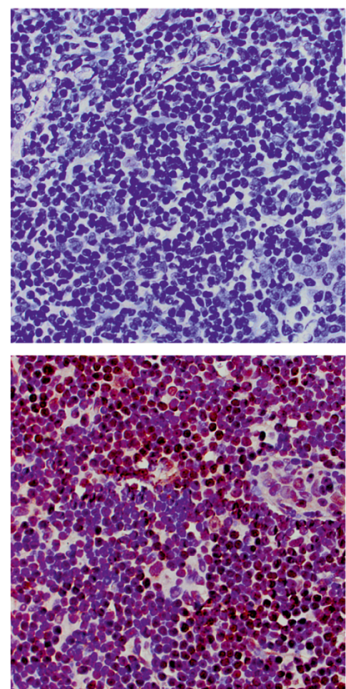


high IgE level, patients showed a limited immune response, and are susceptible to recurrent viral and sinopulmonary infections, atopy and allergic diseases. In some cases, different types of skin cancer and lymphomas have been described [19, 20]. Most patients carrying a DOCK 8 mutation have a decreased number of $\mathrm{CD}^{+}$and $\mathrm{CD}^{+}$T cells $[20,33]$, an observation that runs in parallel to the phenotype of a variable $\mathrm{CD} 3^{+}$ $\mathrm{T}$ cell frequency with a slight reduction more pronounced in $\mathrm{CD}^{+} \mathrm{T}$ cells than in $\mathrm{CD} 8^{+} \mathrm{T}$ cells, observed in peripheral blood of LEW.1AR1-iddm rats [13]. In the present study, we confirmed a variable $\mathrm{CD}^{+}{ }^{+} \mathrm{T}$ cell frequency in all $\mathrm{F} 2$ animals homozygous for the Dock 8 mutation. In addition, Dock8-deficient mice are affected by a decreased number of peripheral $\mathrm{CD}^{+} \mathrm{T}$ cells. In these animals, the development of the T cells is normal but mature single-positive $\mathrm{CD} 4^{+} \mathrm{T}$ cells accumulate in the thymus. The reduced $\mathrm{CD} 4^{+}$and $\mathrm{CD} 8^{+} \mathrm{T}$ cell numbers in peripheral blood are an indication for an increased turnover and a decreased survival rate [33]. Furthermore, DOCK8 has not only an effect on T cells but also on B cells and NK cells [34-37]. These findings are in agreement with those in the LEW.1AR1-iddm rat [13]. Additionally, a decrease of the NKT cell population was observed in the LEW.1AR1-iddm rat, as had been described before already in Dock 8 deficient mice [38]. A defective NKT cell development and a reduced survival rate of $\mathrm{T}$ cells in the circulation have been reported also in Gimap5 (GTPase of the immune associated protein 5) knockout mice [39]. Likewise, the Dock8 mutation in the LEW.1AR1-iddm rat may influence development and survival of the NKT cells through changes in the GTPase activity.

Gene expression analyses in the rat showed that Dock 8 was highly expressed in lymphatic organs in the background strain LEW.1AR1. The mutation in the Dock 8 gene went along with a reduction of the expression level in the LEW.1AR1-iddm rat. Therefore our findings are in agreement with a high gene expression of other DOCK genes, DOCK10 and DOCK11, on human lymphocytes, both $\mathrm{T}$ and $\mathrm{B}$ cells, as well as in lymphatic organs $[40,41]$.

The LEW.1AR1-iddm rat [4], the BB rat [3] and the Komeda rat [42] are rat models for type 1 diabetes. What they have in common is the same MHC II haplotype $B / D^{u}[9$, 42-44], which is a prerequisite for the development of autoimmune diabetes in rats. In addition, we identified in our study in all these rat models the same SNP genotype at position 263, 216, 228 (RGSC Genome Assembly v3.4) within exon 11 of Vwa2 as a factor in the genetic background, which may contribute to disease development.

A distinct mutation in each of the type 1 diabetes rat models is of crucial importance for diabetes susceptibility. The mutation in the Komeda rat is located in the Cblb gene (RNO11), a member of the $\mathrm{Cbl} / \mathrm{Sli}$ family of ubiquitin-protein ligases [42]. Among a number of other functions, Cblb also regulates T cell activation $[45,46]$. The mutation in the BB rat leads to a peripheral lymphopenia and was identified in the Gimap5
(Ian5) gene (RNO4), which encodes a GTPase $[47,48]$. The spontaneous mutation in the LEW.1AR1-iddm rat was identified in the Dock 8 gene. This mutation is accompanied by autoimmune diabetes development and a variable $\mathrm{CD}^{+} \mathrm{T}$ cell frequency in peripheral blood. It is known that other mutations in the Dock family also lead to an imbalance in the immune system in humans [23, 24]. Therefore, the LEW.1AR1-iddm rat is an interesting model to elucidate the importance of GEFs for the function of the different immune cell types and their relationships to the development of autoimmune diabetes in animal models and in humans.

In conclusion, the identification of the mutation in the Dock8 gene of the LEW.1AR1-iddm rat as well as of the single mutations in other genes in the BB [47, 48] and Komeda [42] rat models of human type 1 diabetes show that single mutations contribute to a polygenic threshold required for disease development. Of particular interest in this context are the similarities between BB and LEW.1AR1-iddm rats; in one case the direct loss of the GTPase function and in the other case the loss of function of the regulatory GEF of the GTPase by the respective mutation.

Acknowledgements We are very grateful to A. Bone (School of Pharmacy and Biomolecular Sciences, University of Brighton, Brighton, UK) and Y. Nakaya (Institute of Health Biosciences, Department of Nutrition and Metabolism, University of Tokushima Graduate School, Tokushima, Japan) for providing tissue samples from the BB rat and the Komeda rat. The technical assistance of D. Lischke, C. Salinas (both from the Institute of Clinical Biochemistry, Hannover Medical School, Hannover, Germany) and M. Meyer (Institute for Laboratory Animal Science, Hannover Medical School, Hannover, Germany) is gratefully acknowledged.

Contribution statement TA and AJ planned the study, performed research and analysed the data. SNP data were provided and KASPar analyses were designed, performed and interpreted by EC. Structural analyses were designed, performed and interpreted by GT. The study design was conceived and data acquisition and study execution were supervised by DW, HJH and SL. The manuscript was written by TA, DW, AJ, GT, EC, $\mathrm{HJH}$ and SL. All authors approved the final version. TA is the guarantor of this work.

Funding This work has been supported by a grant from the European Union (Collaborative Project NAIMIT in the 7th Framework Programme, Contract No. 241447) to SL, a grant from the Deutsche Forschungsgemeinschaft (JO 395/2-1) and a grant from the German Foundation 'Das zuckerkranke Kind' to TA.

Duality of interest The authors declare that there is no duality of interest associated with this manuscript.

\section{References}

1. Jörns A, Arndt T, Vilsendorf AM et al (2014) Islet infiltration, cytokine expression and beta cell death in the NOD mouse, BB rat, Komeda rat, LEW.1AR1-iddm rat and humans with type 1 diabetes. Diabetologia 57:512-521 
2. Lally FJ, Bone AJ (2002) Animal models of type 1 diabetes. In: Pickup JC, Williams G (eds) Textbook of diabetes. Blackwell Scientific Publications, Oxford, pp 19.11-19.17

3. Mordes JP, Bortell R, Blankenhorn EP, Rossini AA, Greiner DL (2004) Rat models of type 1 diabetes: genetics, environment, and autoimmunity. Ilar J 45:278-291

4. Lenzen S, Tiedge M, Elsner M et al (2001) The LEW.1AR1/Ztmiddm rat: a new model of spontaneous insulin-dependent diabetes mellitus. Diabetologia 44:1189-1196

5. Arndt T, Wedekind D, Weiss $\mathrm{H}$ et al (2009) Prevention of spontaneous immune-mediated diabetes development in the LEW.1AR1iddm rat by selective $\mathrm{CD} 8^{+} \mathrm{T}$ cell transfer is associated with a cytokine shift in the pancreas-draining lymph nodes. Diabetologia 52:1381-1390

6. Wedekind D, Weiss H, Jörns A, Lenzen S, Tiedge M, Hedrich HJ (2005) Effects of polyinosinic-polycytidylic acid and adoptive transfer of immune cells in the LEW.1AR1-iddm rat and in its coisogenic LEW.1AR1 background strain. Autoimmunity 38:265-275

7. Jörns A, Günther A, Hedrich HJ, Wedekind D, Tiedge M, Lenzen S (2005) Immune cell infiltration, cytokine expression, and beta-cell apoptosis during the development of type 1 diabetes in the spontaneously diabetic LEW.1AR1/Ztm-iddm rat. Diabetes 54:2041-2052

8. Jörns A, Rath KJ, Terbish T et al (2010) Diabetes prevention by immunomodulatory FTY720 treatment in the LEW.1AR1-iddm rat despite immune cell activation. Endocrinology 151:3555-3565

9. Weiss H, Bleich A, Hedrich HJ et al (2005) Genetic analysis of the LEW.1AR1-iddm rat: an animal model for spontaneous diabetes mellitus. Mamm Genome 16:432-441

10. Pickup JC, Williams G (2003) Textbook of diabetes 1. Blackwell, Oxford

11. Ellerman KE, Like AA (2000) Susceptibility to diabetes is widely distributed in normal class IIu haplotype rats. Diabetologia 43:890898

12. Weiss H, Arndt T, Jörns A et al (2008) The mutation of the LEW.1AR1-iddm rat maps to the telomeric end of rat chromosome 1. Mamm Genome 19:292-297

13. Arndt $\mathrm{T}$, Jörns A, Weiss $\mathrm{H}$ et al (2013) A variable $\mathrm{CD}^{+} \mathrm{T}$ cell frequency in peripheral blood lymphocytes associated with type 1 diabetes mellitus development in the LEW.1AR1-iddm rat. PLoS One 8:e64305

14. Nijman IJ, Kuipers S, Verheul M, Guryev V, Cuppen E (2008) A genome-wide SNP panel for mapping and association studies in the rat. BMC Genomics 9:95

15. Harada $Y$, Tanaka $Y$, Terasawa $M$ et al (2012) DOCK8 is a Cde42 activator critical for interstitial dendritic cell migration during immune responses. Blood 119:4451-4461

16. Jörns A, Akin M, Arndt T et al (2014) Anti-TCR therapy combined with fingolimod for reversal of diabetic hyperglycemia by beta cell regeneration in the LEW.1AR1-iddm rat model of type 1 diabetes. J Mol Med (Berl) 92:743-755

17. Ruusala A, Aspenstrom P (2004) Isolation and characterisation of DOCK8, a member of the DOCK180-related regulators of cell morphology. FEBS Lett 572:159-166

18. Yang J, Zhang Z, Roe SM, Marshall CJ, Barford D (2009) Activation of Rho GTPases by DOCK exchange factors is mediated by a nucleotide sensor. Science 325:1398-1402

19. Engelhardt KR, McGhee S, Winkler S et al (2009) Large deletions and point mutations involving the dedicator of cytokinesis 8 (DOCK8) in the autosomal-recessive form of hyper-IgE syndrome. J Allergy Clin Immunol 124(1289-1302):e1284

20. Zhang Q, Davis JC, Lamborn IT et al (2009) Combined immunodeficiency associated with DOCK8 mutations. N Engl J Med 361:2046-2055
21. Zhang Q, Zhou YF, Zhang CZ, Zhang X, Lu C, Springer TA (2009) Structural specializations of A2, a force-sensing domain in the ultralarge vascular protein von Willebrand factor. Proc Natl Acad Sci U S A 106:9226-9231

22. Eller E, Vardi P, Daly MJ et al (2004) IDDM17: polymorphisms in the AMACO gene are associated with dominant protection against type 1A diabetes in a Bedouin Arab family. Ann N Y Acad Sci 1037:145-149

23. Cote JF, Vuori K (2007) GEF what? Dock180 and related proteins help Rac to polarize cells in new ways. Trends Cell Biol $17: 383-393$

24. Su HC (2010) Dedicator of cytokinesis 8 (DOCK8) deficiency Curr Opin Allergy Clin Immunol 10:515-520

25. Mou F, Praskova M, Xia F et al (2012) The Mst1 and Mst2 kinases control activation of rho family GTPases and thymic egress of mature thymocytes. J Exp Med 209:741-759

26. Gadea G, Blangy A (2014) Dock-family exchange factors in cell migration and disease. Eur J Cell Biol 93:466-477

27. Fukui $\mathrm{Y}$, Hashimoto O, Sanui T et al (2001) Haematopoietic cellspecific CDM family protein DOCK2 is essential for lymphocyte migration. Nature 412:826-831

28. Nombela-Arrieta C, Lacalle RA, Montoya MC et al (2004) Differential requirements for DOCK2 and phosphoinositide-3kinase gamma during $\mathrm{T}$ and $\mathrm{B}$ lymphocyte homing. Immunity 21:429-441

29. Nombela-Arrieta C, Mempel TR, Soriano SF et al (2007) A central role for DOCK2 during interstitial lymphocyte motility and sphingosine-1-phosphate-mediated egress. J Exp Med 204:497-510

30. Sanui T, Inayoshi A, Noda M et al (2003) DOCK2 regulates Rac activation and cytoskeletal reorganization through interaction with ELMO1. Blood 102:2948-2950

31. Meller N, Merlot S, Guda C (2005) CZH proteins: a new family of Rho-GEFs. J Cell Sci 118:4937-4946

32. Sinha S, Yang W (2008) Cellular signaling for activation of Rho GTPase Cdc42. Cell Signal 20:1927-1934

33. Lambe $\mathrm{T}$, Crawford $\mathrm{G}$, Johnson AL et al (2011) DOCK8 is essential for $\mathrm{T}$ cell survival and the maintenance of $\mathrm{CD} 8+\mathrm{T}$ cell memory. Eur J Immunol 41:3423-3435

34. Ham H, Guerrier S, Kim J et al (2013) Dedicator of cytokinesis 8 interacts with talin and Wiskott-Aldrich syndrome protein to regulate NK cell cytotoxicity. J Immunol 190:3661-3669

35. Jabara HH, McDonald DR, Janssen E et al (2012) DOCK8 functions as an adaptor that links TLR-MyD88 signaling to B cell activation. Nat Immunol 13:612-620

36. Mizesko MC, Banerjee PP, Monaco-Shawver L et al (2013) Defective actin accumulation impairs human natural killer cell function in patients with dedicator of cytokinesis 8 deficiency. J Allergy Clin Immunol 131:840-848

37. Randall KL, Lambe T, Johnson AL et al (2009) Dock8 mutations cripple B cell immunological synapses, germinal centers and longlived antibody production. Nat Immunol 10:1283-1291

38. Crawford G, Enders A, Gileadi U, Stankovic S, Zhang Q, Lambe T et al (2013) DOCK8 is critical for the survival and function of NKT cells. Blood 122:2052-2061

39. Schulteis RD, Chu H, Dai X et al (2008) Impaired survival of peripheral T cells, disrupted NK/NKT cell development, and liver failure in mice lacking Gimap5. Blood 112:4905-4914

40. Nishikimi A, Meller N, Uekawa N, Isobe K, Schwartz MA, Maruyama M (2005) Zizimin2: a novel, DOCK180-related Cdc42 guanine nucleotide exchange factor expressed predominantly in lymphocytes. FEBS Lett 579:1039-1046

41. Yelo E, Bernardo MV, Gimeno L, Alcaraz-Garcia MJ, Majado MJ, Parrado A (2008) Dock10, a novel CZH protein selectively induced by interleukin-4 in human B lymphocytes. Mol Immunol $45: 3411-3418$ 
42. Yokoi N, Komeda K, Wang HY et al (2002) Cblb is a major susceptibility gene for rat type 1 diabetes mellitus. Nat Genet 31:391-394

43. Jacob HJ, Pettersson A, Wilson D, Mao Y, Lernmark A, Lander ES (1992) Genetic dissection of autoimmune type I diabetes in the BB rat. Nat Genet 2:56-60

44. Kawano K, Hirashima T, Mori S, Saitoh Y, Kurosumi M, Natori T (1991) New inbred strain of Long-Evans Tokushima lean rats with IDDM without lymphopenia. Diabetes 40:1375-1381

45. Fang D, Wang HY, Fang N, Altman Y, Elly C, Liu YC (2001) Cblb, a RING-type E3 ubiquitin ligase, targets phosphatidylinositol 3kinase for ubiquitination in T cells. J Biol Chem 276:4872-4878
46. Zhang J, Bardos T, Li D et al (2002) Cutting edge: regulation of $\mathrm{T}$ cell activation threshold by CD28 costimulation through targeting Cbl-b for ubiquitination. J Immunol 169:2236-2240

47. Krücken J, Schroetel RM, Müller IU et al (2004) Comparative analysis of the human gimap gene cluster encoding a novel GTPase family. Gene 341:291-304

48. MacMurray AJ, Moralejo DH, Kwitek AE et al (2002) Lymphopenia in the BB rat model of type 1 diabetes is due to a mutation in a novel immune-associated nucleotide (Ian)-related gene. Genome Res 12:1029-1039 